Revista Temas Socio Jurídicos

Vol. 37 No 74 Enero - Junio de 2018

pp. $103-115$

ISSN: $0120-8578$

ISSN electrónico: 2590-8901

\title{
LA CONSULTA POPULAR FRENTE A LA INTERVENCIÓN ESTATAL EN MATERIA MINERA
}

Francis Janeth Balaguera Triana ${ }^{1}$

Marid Viviana Bautista Galvis ${ }^{2}$

Sol Yahaira Hernández Duarte ${ }^{3}$

Recibido: 2 de Junio de 2018

Aprobado: 30 de Junio 2018

\begin{abstract}
RESUMEN.
El presente artículo analiza cómo la dinámica interventora del Estado colombiano en las actividades económicas, ha incidido en el incremento de consultas populares en temas mineros. Ésta se presenta como un instrumento mediante el cual la comunidad hace frente al otorgamiento desproporcionado de títulos mineros que no cuentan con el requisito de participación de la comunidad afectada o beneficiada. Además, se proponen estrategias que el Estado debería adoptar para garantizar el bien común y el interés general acorde con los fines constitucionales de guiar la economía bajo parámetros del desarrollo sostenible.
\end{abstract}

Palabras clave: Democracia participativa, intervención estatal en la minería, consulta popular, licencia ambiental, contrato de concesión minera.

1 Estudiante de Derecho, V semestre Universidad Autónoma de Bucaramanga. Semillero de investigación "En pro de una cultura de la integración”. Correo electrónico fbalaguera@ unab.edu.co. Contacto 3183770788

2 Estudiante de Derecho, V semestre Universidad Autónoma de Bucaramanga. Semillero de investigación “En pro de una cultura de la integración”. Correo electrónico mbautista866@ unab.edu.co. Contacto 3156004106

3 Estudiante de Derecho, V semestre Universidad Autónoma de Bucaramanga. Semillero de investigación "En pro de una cultura de la integración”. Correo electrónico Shernandez203@ unab.edu.co. Contacto 3188249333 


\title{
THE PEOPLE'S CONSULTATION AGAINST STATE INTERVENTION IN MINING MATTERS
}

\begin{abstract}
.
The present article analyses how the interventionist dynamic of the Colombian State in economic activities has influence the increase of popular consultations on mining issues. This is presented as an instrument through which the community confronts the disproportionate release of mining titles that do not have the participation requirement of the affected or benefited community. Moreover, there are some strategies proposed that the State should adopt in order to guarantee the common wellbeing and the general interest according to the constitutional purposes of guiding the economy under sustainable development parameters.
\end{abstract}

Key words: Participatory democracy, state Intervention in the mining sector, popular referendum, environmental licensing, mining concession contract.

\section{O REFERENDO CONTRA A INTERVENÇÃO DO ESTADO NA MINERAÇÃO}

\section{RESUMO}

Este artigo analisa como a dinâmica de intervenção do Estado colombiano nas atividades econômicas influenciou $\mathrm{o}$ aumento das consultas populares sobre questões de mineração. O referendo é apresentado como um instrumento através do qual a comunidade enfrenta a concessão desproporcional de títulos de mineração que não tem o requisito de participação da comunidade afetada ou beneficiada. Além disso, inclui propostas estratégicas que o Estado deveria adotar para garantir o bem comum e atender o interesse da população, de acordo com os propósitos constitucionais de orientar a economia sob parâmetros de desenvolvimento sustentável.

Palavras-chave: democracia, intervenção do Estado sobre a mineração, consulta popular, licença ambiental, contrato de concessão mineira.

\section{INTRODUCCIÓN.}

Este artículo hace parte del proyecto de investigación "La consulta popular frente a la intervención estatal en la minería", en el marco del semillero 
"En pro de una Cultura de la Integración" del Centro de Estudios Latinoamericanos, adscrito al Programa de Derecho de la Universidad Autónoma de Bucaramanga, UNAB.

La riqueza que posee Colombia de recursos minerales y el auge en los precios internacionales del mercado minero han propiciado la implementación de normas y políticas públicas que apuntan al fomento de la actividad extractiva de los recursos mineros, en ese contexto se expide el código de minas, Ley 685 de 2001, el cual otorga el carácter de utilidad pública e interés social a la actividad minera en todas sus fases, aspecto que le reconoce una protección especial.

En este marco, el desarrollo del sector minero presentó un incremento debido al auge de inversión extranjera y nacional en el sector, dejándole al Estado beneficios económicos provenientes de impuestos y regalías proporcionales a los precios de las materias primas y a la cantidad extraída. Sin embargo, el costo de transacción de los proyectos mineros deja, en las comunidades donde se ejecuta, consecuencias ambientales, sociales, económicas y políticas.

En principio, dichos afectaciones deberían minimizarse con la acción del Estado, puesto que, es función de él intervenir en pro del interés general, de tal manera que logre encontrar un equilibrio entre esta actividad económica y el desarrollo sostenible, para ello, cuenta con mecanismos que tienen por objeto impedir, mitigar o eliminar los efectos negativos de la minería, no obstante, la eficacia de los mismos se pone en duda en la realidad, lo que contribuye a que la comunidad recurra a otros medios, como la consulta popular, que si les garanticen medidas eficaces y concretas para la protección de sus derechos individuales y colectivos, que en ocasiones buscan, incluso, frenar proyectos mineros, derivando en un "boom de consultas populares".

Debido al aumento en el uso de este mecanismo se han presentado argumentos con los cuales se pretenden desconocer la procedencia y obligatoriedad de los resultados de las consultas. Así, el presente trabajo permitiría mostrar que más que un problema sobre procedencia de la consulta popular en temas mineros o de oposición a la minería en sus territorios, dicho auge debe verse como un llamado de atención a la ineficaz intervención del Estado.

Por ello, la presente investigación tiene como objetivo analizar la relación existente entre la intervención estatal y el auge de las consultas populares en materia minera y para efectos metodológicos, con el fin de responder a la pregunta acerca de ¿Cuál es la relación existente entre la intervención estatal en la minería y el "boom de las consultas populares"? El abordaje de este problema se justifica en la medida de que la discusión sobre el tema minero ocupa la agenda de la discusión en distintos estamentos de la administración del Estado e incide de manera notoria en el desarrollo de la vida cotidiana de las comunidades afectadas. 
El avance que se presenta permite concluir que debe fortalecerse la intervención del Estado en materia minera con el fin de garantizar la armonización de los principios económicos y del interés general.

Para llegar a la presentación de resultados el problema se abordó desde una perspectiva metodológica de tipo mixto (cualitativo y cuantitativo) y desde un método hipotético deductivo a partir de fuentes documentales.

El texto se desarrollará en cuatro secciones:

En la primera, se hace referencia a la promulgación de la Constitución Política de 1991 y a la trascendencia que esta asigna a los nuevos pilares que se establecen en torno a la intervención estatal en la economía, con el fin de lograr la garantía de un orden económico, político y social justo.

En la segunda, se describen aspectos teóricos de la intervención estatal en los diversos sectores de la economía, específicamente en el sector de la explotación de los recursos naturales, en lo concerniente a los mecanismos con los que cuentan las autoridades para cumplir dicho cometido y los vacíos que se derivan del mismo.

En la tercera, se aborda la procedencia y alcance de la consulta popular y se conceptualiza el fenómeno del "boom de las consultas populares".

Y en la cuarta, se presentan unas recomendaciones de cierre a la luz del conflicto analizado que permiten al Estado Social de Derecho el cumplimiento de sus fines, con base en la Constitución Política de 1991.

\section{PROBLEMA DE INVESTIGACIÓN.}

¿Cuál es la relación existente entre la intervención estatal en la minería y el "boom de las consultas populares"?

Frente al problema de investigación se encuentra que hay una relación de causa-efecto, puesto que, el uso de la consulta popular en materia minera es un mecanismo para hacer frente a la ineficaz intervención del Estado en dicha materia.

\section{METODOLOGÍA.}

En el desarrollo de la presente investigación, se hace uso del método hipotético- deductivo en la medida que se parte de un hecho social como objeto de estudio, tal y como lo es "el boom de las consultas populares" en materia minera para darle explicación al mismo por medio de la construcción de una hipótesis que será la que oriente de manera sólida la investigación esclareciendo los fenómenos que dan origen al hecho. En forma conjunta, se utiliza el tipo de investigación científica confirmatoria, apoyada en las técnicas de investigación cualitativa y cuantitativa, en lo correspondiente a que por medio del análisis y comprobación de datos a través de cifras numéricas y la observación natural de los hechos sin ninguna medición, se aportan argumentos que contribuyen a fundamentar 
la validez de la hipótesis planteada de relación causa y efecto que existe entre la multiplicidad de consultas populares y la intervención estatal en la minería.

La presente investigación busca esclarecer la relación existente entre la intervención estatal y el "boom de las consultas populares" en materia minera y por tanto, la metodología que sigue consiste en el análisis de las disposiciones constitucionales, legales y jurisprudenciales que enmarcan dicha relación. Asimismo, se realiza una revisión bibliográfica de libros, informes técnicos e institucionales y de noticias publicadas al respecto.

\section{AVANCES, RESULTADOS Y DISCUSIÓN.}

A continuación, se presenta un avance del trabajo realizado. Por su orden se aborda el texto constitucional en materia de intervención estatal, posteriormente se hace referencia al papel del Estado en la economía y finalmente, al boom de las consultas populares como manifestación de la voluntad de la comunidad.

\subsection{La intervención estatal en la Constitución Política}

La historia ha demostrado que la intervención estatal es necesaria para regular los fenómenos económicos. Colombia desde 1936 acogió esta concepción, por medio del Acto Legislativo número 1 del mismo año, y con disposiciones legislativas posteriores reguló la intervención del Estado en diferentes sectores económicos (Restrepo Medina, 2010). Sin embargo, con la promulgación de la Constitución política de 1991 y con la adopción de un Estado Social de Derecho, se da un giro en los parámetros que enmarcan la intervención estatal, puesto que se adopta a la libertad económica y a la iniciativa privada como pilares de la nueva economía social de mercado, los cuales encuentran un límite en el bien común y el interés general. Dicho límite es la justificación para que el Estado intervenga por medio de las actuaciones de los poderes públicos y busque la armonización del bien común con los principios económicos.

Es así como la intervención del Estado en la economía no corresponde a una sola institución, sino que debe ejercerse concatenadamente entre las diferentes ramas del poder público, puesto que busca conciliar los diferentes intereses que se desprendan de la actividad económica, sin hacer prevalecer los unos sobre otros salvo que medie razón suficiente.

\subsection{La intervención del Estado en la economía}

La intervención del Estado en la economía es ejercida por distintos organismos del Estado en sus respectivos niveles, empero, para efectos metodológicos la intervención se clasifica o se manifiesta de manera global, sectorial y particular. (Corte Constitucional, C- 150/2003)

Es global, cuando versa sobre los pilares del modelo económico in totum, es decir, la libertad económica y la iniciativa privada. 
Es sectorial, si recae en una determinada área, como, por ejemplo, la regulación que debe hacerse en los proyectos de explotación de los recursos naturales no renovables y en las contraprestaciones que estos generen, en pro de preservar el medio ambiente.

Y es particular, si apunta a una cierta situación, como cuando el Estado revoca los derechos adquiridos por un título minero dado el incumplimiento de lo pactado.

En este sentido, las licencias ambientales y los contratos de concesión minera son por antonomasia el reflejo de la intervención estatal en la minería, al ser los principales títulos otorgados por las autoridades en el marco de una actividad extractivita, como lo es la minería.

Las licencias ambientales son un prerrequisito para la etapa de explotación del proyecto minero y constituyen una garantía para que la actividad se ejecute con base en el desarrollo sostenible. Por ello, para su otorgamiento, se ha de evaluar previamente el estudio de impacto ambiental. ${ }^{4}$ Según el artículo 204 del código de minas vigente, este documento debe contener las medidas de contingencia diseñadas por el titular del proyecto, la prueba de la información proporcionada a la comunidad acerca del alcance del plan a realizar y la constancia de haber tomado en consideración las propuestas de aquélla, garantizando así, la preservación del medio ambiente y la participación de las comunidades que se verán afectadas por el proyecto.

A pesar de ello, el desarrollo actual del estudio de impacto ambiental no responde a las exigencias que demanda la actividad minera, tal y como dan cuenta las solicitudes de las licencias ambientales presentadas en el período 2000-2013, de las que, de un total de 6.514 solicitudes, solo el $10.5 \%$ cumplía con el requisito de participación de las comunidades. Asimismo, dentro de este 10,5\% se hizo uso de la figura del tercer interviniente ${ }^{5}$ con un $6,3 \%$ y tan solo en un $2.5 \%$ y en $1.7 \%$ se recurrió a las consultas previas y audiencias públicas, respectivamente. (Defensoría del pueblo, 2015)

Los contratos de concesión minera son aquellos que se celebran entre el Estado y un particular, mediante los cuales se le otorga la facultad a este último, para que efectúe estudios, trabajos y obras de exploración de minerales, en determinadas zonas de propiedad del estatal y en contraprestación a través de regalías u otros peculios, regresa un porcentaje

4 Según el decreto 2041/2014 es el instrumento básico para la toma de decisiones sobre los proyectos, obras o actividades que requieren licencia ambiental.

5 El artículo 69 de la ley 99 de 1993 define la figura del tercer interviniente como el derecho que tiene cualquier persona natural o jurídica o privada, sin necesidad de demostrar interés jurídico alguno, a intervenir en las actuaciones administrativas iniciadas para la expedición, modificación o cancelación de permisos o licencias de actividades que afecten o puedan afectar el medio ambiente o para la imposición o revocación de sanciones por el incumplimiento de las normas y regulaciones ambientales. 
de sus utilidades al Estado, sin que ello implique la transferencia del dominio del terreno. Este tipo de contrato tiene unas características propias y un objeto que lo identifica, el cual consiste en que el particular contratista debe en mayor medida procurar impedir, eliminar o mitigar el impacto de la actividad minera autorizada.

Sin embargo, el cumplimiento de este objeto se está viendo truncado, puesto que, para el mes de abril del año 2015 se encontraban vigentes 9.594 títulos, 5.084.084 ha, correspondientes al 4,5\% de los 114 millones de hectáreas del territorio nacional, de los cuales 3.557 estaban en etapa de exploración, 2.273 en la fase de construcción y montaje y 3.764 en la etapa de explotación. Adicionalmente, de los más de 9.500 vigentes en el país, el $78 \%$ de los títulos, es decir 7.483 , tienen algún tipo de incumplimiento y dentro de estos, en 350 títulos se encontró que no se contaba con licencia ambiental y, aun así, estaban explotando. (Defensoría del pueblo, 2015)

Lo anterior evidencia, primero, la falta de rigurosidad al momento de regular las diferentes etapas del proyecto extractivo y segundo, que el Estado no tiene el conocimiento adecuado del territorio sujeto a la utilización para la minería, lo que genera una carencia de planificación en el manejo de los recursos naturales contrariando así el mandato constitucional del artículo 80, y generando una entrega desproporcionada de los títulos mineros, tal y como las cifras lo demuestran.

\subsection{El boom de las consultas populares como manifestación de la voluntad de la comunidad.}

La ausencia de una intervención integral por parte del Estado ha generado lo que los medios han denominado como "El boom de las consultas populares", en otras palabras, la consulta popular es una válvula de escape para los habitantes de los municipios, quienes son los afectados directamente por la entrega indiscriminada de títulos mineros. Estos pronunciamientos no solo tienen como finalidad oponerse a la minería, sino que también deben verse como un llamado de atención a la ineficaz intervención del Estado para que los requisitos que permitan el desarrollo de la misma sean más contundentes y rigurosos.

Sin embargo, se ha puesto en tela de juicio la procedencia de estas consultas a nivel local, por la connotación económica de la minería, los costos por la realización de la consulta y los requisitos mismos frente al alcance de la consulta popular.

El aporte de la minería a la economía colombiana, según el documento de la Política Minera Nacional (2016), representó entre 2010 y 2015 un promedio del $2.2 \%$ del PIB y en lo referente a las exportaciones y a la inversión extranjera, los aportes fueron del $19.6 \%$ y $16 \%$ respectivamente. Además, contribuye a las finanzas públicas con impuestos de renta, patrimonio, predial, ICA e IVA, así como con las regalías. Incluso, solo con los recursos obtenidos por regalías entre este mismo período, que 
ascienden a $\$ 9.7$ billones, se pueden financiar dos años completos del programa social Familias en Acción. Adicionalmente, esta actividad genera en promedio 215.000 empleos anuales. Situando a la minería en un tema de orden nacional.

De igual forma, la importancia de la minería en el país, ha sido constatada por la UPME, que en el año 2015 certificó el potencial que entre los diferentes minerales que tiene Colombia, el carbón térmico y metalúrgico coloca al país como el principal productor de carbón de Sur América, el cuarto país exportador de este mineral y el undécimo productor en el mundo, lo que lo convierte en un país tomador de precios internacionales. (Ministerio de Minas, 2016)

Respecto al alcance, tanto a nivel nacional como territorial, las consultas podrán versar sobre todos los ámbitos de la vida individual, familiar, social y comunitaria, pero, su procedencia estará limitada a las competencias de quien la convoque o de la esfera donde se lleve a cabo. (Corte Constitucional, C-150/2015)

Lo anterior puede poner en tela de juicio la viabilidad de la consulta popular a nivel local, porque, en principio, se podría deducir que en lo referente a minería la competencia es de índole nacional, teniendo en cuenta que los calificados para autorizar la etapa de explotación de un proyecto minero, por medio de la concesión de licencias ambientales, son el Ministerio de Medio Ambiente y Desarrollo Sostenible, la Autoridad Nacional de Licencias Ambientales (ANLA) y las Corporaciones Autónomas Regionales en las situaciones establecidas en la ley general ambiental.

Como un ejemplo de este aspecto, la Corte Constitucional en sentencia T-123/09 considera como acertada la negativa de la Corporación Autónoma Regional de Cundinamarca para desatender el carácter vinculante de la consulta popular adelantada en el municipio de Nemocón, puesto que las decisiones que debe adoptar esta entidad en materia ambiental "trascienden la esfera estrictamente municipal y se proyectan en un escenario regional de mayor envergadura". (Corte Constitucional, $\mathrm{T}-123 / 09)$

Sin embargo, la Ley 136 de 1994 es clara al respecto, al imponer de manera expresa a los municipios la obligación de realizar una consulta cuando el desarrollo de un proyecto de naturaleza turística, minera o de otro tipo amenace producir un cambio significativo en el uso del suelo, que dé lugar a una transformación en las actividades tradicionales de un municipio, disposición que se fundamenta en la facultad de los mismos para reglamentar los usos del suelo, acorde con lo establecido en la Constitución Política en el artículo 313 \#7. En ese orden de ideas, la consulta popular es la opinión que una determinada autoridad solicita a la ciudadanía sobre un aspecto específico de interés nacional, regional o local, que, dada su naturaleza de derecho fundamental, sus resultados adoptan un carácter obligatorio que conlleva traducirlos en acciones 
De lo expuesto hasta el momento es posible visibilizar la tensión que surge entre la competencia privativa del Estado para otorgar y revocar títulos mineros frente al derecho de las entidades territoriales a convocar a la comunidad para decidir sobre el uso de sus suelos. No obstante, "el concepto de unidad del Estado colombiano no puede ser utilizado como pretexto para desconocer la capacidad de autogestión de las entidades territoriales, y a su turno, la autonomía de las entidades territoriales no puede ser entendida de manera omnímoda, hasta el punto de hacer nugatorias las competencias naturales de las instancias territoriales centrales." (Corte Constitucional, C-145/2015).

Por lo tanto, para no menoscabar ni un extremo ni otro, se debe entender la unidad del Estado y la autonomía de los entes territoriales en conjunto, es decir, que el Estado no debería iniciar proyectos mineros desconociendo la voluntad de la comunidad, ni ella puede desconocer los lineamientos establecidos a nivel nacional.

\section{CONCLUSIONES}

La intervención del Estado en la economía es una potestad que indudablemente repercute en el ámbito social, por ende, es necesaria para armonizar el bien común con los principios económicos. Aunque las cifras presentadas en la investigación revelan una insuficiencia en la intervención del Estado en materia minera y poder cumplir la finalidad para la cual fue instituida, ello no debe traducirse en un modelo económico que carezca de control estatal, sino que se constituye en un supuesto para reformarla. $\mathrm{Su}$ reestructuración debe estar encaminada a dar cabal cumplimiento a los fines constitucionales de guiar la economía sin sobrepasar los límites de la protección ambiental.

En ese orden de ideas, es menester que exista una veeduría más rigurosa por parte del Estado en la actividad minería que tenga como criterio base el desarrollo sostenible y se materialice en una planificación en materia territorial que permita identificar y clasificar las áreas de potencial extracción minera para que los títulos mineros dejen de otorgarse de manera desproporcionada y se genere un cambio cuantitativo en los títulos que se autoricen para explorar y explotar.

Frente a las licencias ambientales, es necesario que dejen de otorgarse como una simple directriz nacional y se tenga en cuenta la participación de la población potencialmente afectada o beneficiada. Es decir, que al momento de evaluar la autoridad el estudio de impacto ambiental, tenga en cuenta dos criterios que serían determinantes para la entrega o no de la licencia, el primero, que se realice la socialización del proyecto minero y que la información proporcionada sea de fondo, y el segundo, que se conjuguen las modalidades utilizadas para la socialización, es decir, que además de una audiencia pública se realice una consulta popular. 
La ausencia de una intervención integral por parte del Estado en materia minera, como se expresó en el desarrollo del artículo, ha presentado a la consulta popular como una válvula de escape para los habitantes de los municipios donde se ejecutan o van a ejecutar proyectos de explotación minera, sin embargo, la respuesta a estos pronunciamientos ha sido el desconocimiento de los mismos por argumentos económicos que demuestran que la minería hoy en día se constituye en una importante fuente económica por lo que debería ser manejada por las autoridades nacionales, pero que no pueden ser óbices para desconocer la manifestación popular y las afectaciones ecológicas que se generan en el medio ambiente, porque es un garantía del "ser humano vivir e interactuar dentro de un medio ambiente sano que le permita desarrollar su existencia en condiciones dignas, sin que este último esté amenazado por la actividad extractiva estatal." (Corte Constitucional, T-622-16)

En cuanto a la tensión que surge entre la competencia privativa del Estado para otorgar y revocar títulos mineros frente al derecho de las entidades territoriales a convocar a la comunidad para decidir sobre el uso de sus suelos, si bien hay una confusión normativa frente a quién es el competente, no es un problema de normas sino de que las actuaciones de las autoridades estén precedidas por la garantía al interés general, la participación popular y la preservación del medio ambiente.

Esto permitiría lograr la materialización de las cláusulas del Estado Social de Derecho y que no queden en una mera eficacia simbólica.

\section{REFERENCIAS BIBLIOGRÁFICAS}

Cabrera, M., Espitia J., Fierro J., Negrete R., Pardo L., Rudas G., \& Vargas F. (2013). Minería en Colombia; Fundamentos para superar el modelo extractivista. Recuperado de https://redjusticiaambientalcolombia. files.wordpress.com/2013/05/mineria-en-colombia-fundamentospara-superar-el-modelo-extractivista2013.pdf

Código de Minas Colombiano [CMC]. Ley 685 de 2001. Agosto 15 de 2001. (Colombia).

COLPRENSA., (Junio 11 de 2017) ¿Las consultas contra minería y petróleo amenazan la economía? El colombiano. Recuperado de http://www. elcolombiano.com/negocios/economia/consultas-popularescontra-mineria-y-petroleo-e-impacto-en-la-inversion-en-colombiaNA6705168

Constitución Política de Colombia [Const]. Julio 7 de 1991 (Colombia).

Corte Constitucional de Colombia. Sentencia C- 150/03. (MP. Manuel José Cepeda Espinosa: Febrero 25 de 2003).

Corte Constitucional de Colombia. Sentencia C-035/16. (MP. Gloria 
Stella Ortiz Delgado: Febrero 8 de 2016).

Corte Constitucional de Colombia. Sentencia C-123/14. (MP. Alberto Rojas Ríos: Marzo 5 de 2014).

Corte Constitucional de Colombia. Sentencia C-145/15. (MP. Martha Victoria Sáchica Méndez: Abril 6 de 2015).

Corte Constitucional de Colombia. Sentencia C-150/15. (MP. Mauricio González Cuervo: Abril 8 de 2015).

Corte Constitucional de Colombia. Sentencia C-228/10. (MP. Luis Ernesto Vargas Silva. Marzo 24 de 2010).

Corte Constitucional de Colombia. Sentencia C-389/16. (MP. María Victoria Calle Correa: Julio 27 de 2016).

Corte Constitucional de Colombia. Sentencia C-567/06. (MP. Eduardo Cifuentes: Enero 27 de 2006).

Corte Constitucional de Colombia. Sentencia T-123/09. (MP. Clara Inés Vargas Hernández: Febrero 24 de 2009).

Corte Constitucional de Colombia. Sentencia T-445/16. (MP. Jorge Iván Palacio Palacio: Agosto 19 de 2016).

Corte Constitucional de Colombia. Sentencia T-622/16. (MP. Jorge Iván Palacio Palacio: Noviembre 10 de 2016).

Decreto 3573 de 2011. Por el cual se crea la Autoridad Nacional de Licencias Ambientales -ANLA- y se dictan otras disposiciones. Septiembre 27 de 2011.

Defensoría del Pueblo. La minería sin control, un enfoque desde la vulneración de los DD. HH, 2015. Bogotá, D.C., Colombia. Recuperado de http:// www.defensoria.gov.co/public/pdf/InformedeMinerIa2016.pdf

Dietz, K., (2018.) Consultas populares mineras en Colombia: Condiciones de su realización y significados políticos. El caso de La Colosa. Colombia Internacional (93): 93-117. DOI: https://dx.doi.org/10.7440/ colombiaint93.2018.04

Gonzales, J., (Marzo 22 de 2018) En 2017 se realizaron 7 consultas populares y hay 54 pendientes. La República. Recuperado de https://www. larepublica.co/especiales/minas-y-energia/en-2017-se-realizaron7-consultas-populares-y-hay-54-pendientes-2613185

Jáuregui, D., (Abril 12 de 2018) La Corte Constitucional decidirá cuál será el futuro de las consultas mineras. Asuntos legales. Recuperado de https:// 
www.asuntoslegales.com.co/actualidad/corte-constitucionaldecidira-el-futuro-de-las-consultas-mineras-2713078

Ley 134 de 1994. Por la cual se dictan normas sobre mecanismos de participación ciudadana. Mayo 31 de 1994. DO. No. 41373.

Ley 136 de 1994. Por la cual se dictan normas tendientes a modernizar la organización y el funcionamiento de los municipios. Junio 02 de 1994. DO. No. 41.377.

Ley 1757 de 2015. Por la cual se dictan disposiciones en materia de promoción y protección del derecho a la participación democrática. Julio 6 de 2015. DO. No. 49565.

Ley 388 de 1997. Por la cual se modifica la Ley 9 de 1989, y la Ley 2 de 1991 y se dictan otras disposiciones. Julio 18 de 1997. DO. No. 43.091 .

Ley 99 de 1993. Por la cual se crea el Ministerio del Medio Ambiente, se reordena el Sector Público encargado de la gestión y conservación del medio ambiente y los recursos naturales renovables, se organiza el Sistema Nacional Ambiental, SINA, y se dictan otras disposiciones. Diciembre 22 de 1993. DO. No. 41146.

Ministerio de Minas. Política Minera de Colombia; Bases para la minería del futuro, 2016. Bogotá, D.C., Colombia. Recuperado de https://www.minminas.gov.co/documents/10180/698204/ Pol\%C3\%ADtica + Minera + de + Colombia + final.pdf/c7b3fcad76da-41ca-8b11-2b82c0671320

Restrepo M., (2010). Colombia. Redefinición normativa de la regulación y el control de la actividad económica en el caso colombiano. Universidad del Rosario. Recuperado de http://www.scielo.org.co/pdf/vniv/n121/ n121a11.pdf

Restrepo, J., \& Torres, J. (2007) Colombia. Análisis sobre el proyecto de ley 010/07 Senado "por el cual se modifica la ley 685 de 2011, Código de Minas. Contraloría delegada para el sector de minas y energía. Recuperado de http://www.senado.gov.co/attachments/117_analisis_2_de_ contraloria_al_codigo_de_minas.pdf

Rodríguez, C., (2015) Colombia. Las nuevas fronteras de la justicia constitucional: la democracia ambiental y las consultas populares en conflictos socioambientales. (Memorias del encuentro constitucional por la tierra). Corte Constitucional de Colombia.

Rodríguez, D., (Abril 13 de 2018) Es legal y constitucional que municipios convoquen a consultas populares sobre minería e hidrocarburos. 
Dejusticia. Recuperado de https://www.dejusticia.org/es-legal-yconstitucional-que-municipios-convoquen-a-consultas-popularessobre-mineria-e-hidrocarburos/

Rojas Díaz, D. (2013). Licencias ambientales en Colombia: límites o autorizaciones para el uso de los recursos naturales. (Tesis de maestría, Universidad Nacional de Colombia). Recuperado dehttp://www.bdigital.unal. edu.co/39891/1/6699693.2013.pdf

Rudas G., Espitia J., Mena J., Pardo L., Fierro J., Olivero J., Caballero K., Guerrero A., Vargas F., Negrete R. (2013) Minería en Colombia; Institucionalidad $y$ territorio, paradojas $y$ conflictos. Recuperado de https://redjusticiaambientalcolombia.files.wordpress. com/2014/01/mineria-en-colombia-contraloria-vol-ii.pdf

Sánchez, V., (Octubre 09 de 2015) Consulta Popular: la decisión del pueblo es obligatoria. Revista Semillas. Recuperado de http://www.semillas. org.co/es/consulta-popular-la-decisi

Silva, S., (Octubre 1 de 2017) Consultas populares, ¿̇por qué el malestar? El Espectador. Recuperado de https://www.elespectador.com/ noticias/medio-ambiente/consultas-populares-por-que-elmalestar-articulo-715906

Uprimny, R., \& Rojas, N. (2015) Colombia. Constitución, industrias extractivas y territorios. (Memorias del encuentro constitucional por la tierra). Corte Constitucional de Colombia. 